\title{
Influência dos Custos de Controle nos Custos das falhas Internas e Externas
}

Juliane Andressa Pavão
Doutorado em Contabilidade em andamento pela Universidade Federal do Paraná -
UFPR

Professora na Universidade Estadual de Maringá - UEM Rua Afonso Pena, 130. Zona 1. Cianorte/PR. CEP: 87200-000

E-mail: julianepavao@hotmail.com

Simone Bernardes Voese

Pós-doutorado em Administração pela Universidade Federal do Mato Grosso do Sul -

UFMS

Professora na Universidade Federal do Paraná - UFPR

Av. Prefeito Lothario Meissner, 632. Jardim Botânico. Curitiba/PR. CEP: 80210-170

E-mail:simone.voese@gmail.com.br

Reinaldo Rodrigues Camacho Doutorado em Controladoria e Contabilidade pela Universidade de São Paulo - USP

Professor na Universidade Estadual de Maringá - UEM Rua Afonso Pena, 130. Zona 1. Cianorte/PR. CEP: 87200-000

E-mail: rrcamacho@usp.br

\section{RESUMO}

O estudo tem como objetivo analisar a influência de custos de prevenção e avaliação na redução de custos de falhas internas e externas no setor de confecções. Trata-se de um estudo quantitativo e descritivo, que teve como estratégia de pesquisa o levantamento e coleta de dados por meio de questionário. Obteve-se 121 respostas que foram analisadas por meio de Análise Fatorial Exploratória (AFE) e Regressão Linear Múltipla com bootstrapping. O principal achado da pesquisa foi que investir no treinamento de funcionários, na manutenção preventiva de máquinas e equipamentos, no desenvolvimento e auditar um sistema de qualidade, isso influencia na redução dos custos de falhas. Porém, a inspeção de materiais comprados e a avaliação da conservação dos materiais em estoque apresentaram comportamento significativo contrário, ocasionando assim, os custos de falhas. Conclui-se que, no setor de confecções, segundo a amostra investigada, os custos de prevenção auxiliam na redução das falhas, mas os custos de avaliação não apresentaram o mesmo comportamento, ressaltando a necessidade de mais pesquisas para compreender essa relação.

Palavras-chave: Custos da Qualidade. Prevenção. Avaliação. Falhas. Confecções. 
Influência dos Custos de Controle nos Custos das falhas Internas e Externas

Juliane Andressa Pavão, Simone Bernardes Voese, Reinaldo Rodrigues Camacho

\title{
Influence of Control Costs on Internal and External Failure Costs
}

\begin{abstract}
The objective of the study is to analyze the influence of prevention and evaluation costs aiming to have the costs of internal and external failures in the clothing sector reduced. It is about a quantitative and descriptive study, that made use of a questionnaire as research strategy to survey and collect data. A total of 121 responses were obtained, which were analyzed by means of Exploratory Factor Analysis (EFA) and Multiple Linear Regression with bootstrapping. The main findings of the research are that to invest in training employees, as well as in preventive maintenance of machines and equipment, and to develop a quality system auditing influences the reduction of failure costs. However, the inspection of purchased materials and the assessment of the conservation of materials in stock showed a significant opposite behavior, causing failure costs. It is concluded that, in the clothing sector, according to the investigated sample, the prevention costs help to reduce failures, but the evaluation costs did not show the same behavior, highlighting the need for more research to understand this relationship.
\end{abstract}

Keywords: Quality Costs. Prevention. Evaluation. Failures. Clothing sector.

\section{Influencia de los Costos de Control en los Costos de fallas Internas y Externas}

\section{RESUMEN}

El estudio tiene como objetivo analizar la influencia de los costos de prevención y evaluación en la reducción de los costos de fallas internas y externas en el sector de la confección. Se trata de un estudio cuantitativo y descriptivo, cuya estrategia de investigación fue relevar y recolectar datos a través de un cuestionario. Se obtuvieron y analizaron 121 respuestas utilizando Análisis Factorial Exploratorio (AFE) y Regresión Lineal Múltiple con bootstraping. El principal hallazgo de la investigación fue que invertir en capacitación de empleados, mantenimiento preventivo de máquinas y equipos, en el desarrollo y auditar un sistema de calidad, eso influye en la reducción de costos de falla. Sin embargo, la inspección de los materiales comprados y la evaluación de la conservación de los materiales en stock mostraron un comportamiento opuesto significativo, provocando costos de falla. Se concluye que, en el sector de la confección, según la muestra investigada, los costos de prevención ayudan a reducir las fallas, pero los costos de evaluación no mostraron el mismo comportamiento, enfatizando la necesidad de más investigación para entender esta relación.

Palabras-clave: Costos de la Calidad. Prevención. Evaluación. Fracasos. Ropa. 
Influência dos Custos de Controle nos Custos das falhas Internas e Externas

Juliane Andressa Pavão, Simone Bernardes Voese, Reinaldo Rodrigues Camacho

\section{INTRODUÇÃO}

As pressões competitivas enfrentadas pelas empresas induzem a uma dependência de melhorias baseadas em resultados e orientadas para a qualidade (Abdelsalam \& Gad, 2009). Os custos da qualidade são custos associados com a definição, criação e controle da qualidade, assim como a avaliação e retroalimentação da conformação da qualidade, e os custos de falhas são aqueles associados aos requisitos de produção e depois que o produto já se encontra com o cliente (Feigenbaum, 1994). Dessa forma, o objetivo da gestão dos Custos da Qualidade (CQ) não é apenas alcançar um produto de boa qualidade atendendo aos requisitos do cliente, mas também fazê-lo com o menor custo (Crosby, 1979; Feigenbaum, 1994; Sakurai, 1997; Abdelsalam \& Gad, 2009).

Estudos investigam a mensuração e avaliação dos CQ no setor de construção civil no Brasil (Maldaner, 2003; Morgan \& Ramos, 2008; Luz, 2011) e em Dubai (Abdelsalam \& Gad, 2009), ou ainda, em indústrias de transformação no Brasil (Sá, 2003) e em manufaturas da Alemanha (Plewa, Kaiser \& Hartmann, 2016). Empresas certificadas pelo Prêmio Nacional de Qualidade (PNQ) também tem sido foco de pesquisas como Alencar e Guerreiro (2004) e Souza e Collaziol (2006). Já Mattos e Toledo (1998) investigaram a respeito da implantação de sistema de CQ.

Os achados de Abdelsalam e Gad (2009) e Plewa, Kaiser e Hartmann (2016) se contrapõem na medida em que Abdelsalam e Gad (2009) encontraram uma correlação positiva entre os custos de prevenção e avaliação e os custos de falhas, ou seja, quanto maiores os custos de prevenção e avaliação, maiores são também os custos de falhas, o que contesta a literatura existente sobre os CQ. Porém, Plewa, Kaiser e Hartmann (2016) identificaram que baixos custos de falhas estão correlacionados com altos custos de prevenção e avaliação.

A motivação para este estudo consiste em investigar se é melhor prevenir do que remediar. Ou seja, qual a influência da ocorrência de custos de prevenção e avaliação na redução dos custos de falhas? Assim, esta pesquisa tem como objetivo analisar a 
influência de custos de prevenção e avaliação na redução de custos de falhas no setor de confecções.

Esta pesquisa possui enfoque no setor de confecções dos municípios de Cianorte e Maringá, Estado do Paraná, no Brasil, devido a importância da qualidade para evitar peças com defeitos, sobras de materiais, retrabalhos, entre outras falhas. E, ainda, essas respectivas regiões destacam-se nacionalmente como o segundo maior polo confeccionista do Brasil, conhecido por fazer parte do "Corredor da Moda" (Carreira, 2001). O estudo desse setor é relevante devido aos problemas que tem enfrentado com o aumento dos produtos importados, principalmente da China.

Este estudo contribui com a literatura pelo fato de testar de forma quantitativa o pressuposto pelos principais autores dos $C Q$ de que investir em custos com prevenção e avaliação causa consequentemente a redução de custos de falhas internas e externas, uma vez que são escassas as pesquisas que investigam essa relação.

Além dessa introdução, a pesquisa apresenta outras quatro seções. A segunda seção aborda a conceituação dos Custos da Qualidade, como também, os estudos anteriores sobre o tema. A terceira seção apresenta os procedimentos metodológicos adotados na realização da pesquisa. A quarta seção apresenta os resultados e discute os achados do estudo. Por fim, a última seção apresenta as conclusões.

\section{REFERENCIAL TEÓRICO}

Neste tópico será tratada a plataforma teórica necessária para compreensão do objeto de estudo. Dessa forma está constituído em três partes: Custos da qualidade, definições e classificações; Estudos relacionados, apresentando os achados dos principais estudos sobre o tema, e ainda, Hipóteses teórico-empírica da pesquisa, a qual apresenta as hipóteses que serão testadas. 
Influência dos Custos de Controle nos Custos das falhas Internas e Externas Juliane Andressa Pavão, Simone Bernardes Voese, Reinaldo Rodrigues Camacho

\subsection{Custos da Qualidade}

São muitas as definições de qualidade encontradas na literatura (Feigenbaum, 1994; Sakurai, 1997; Deming, 2003; Juran, 2009). Dentre elas, a definição de qualidade é a característica principal de um produto. Para os clientes, quanto melhores as características de um produto e menor deficiência, mais alta é a sua qualidade (Juran, 2009). Porém, a palavra qualidade não significa "melhor" em sentido amplo. No contexto organizacional, significa buscar satisfazer as condições estabelecidas e valorizadas pelos clientes, independentemente do produto ser tangível ou intangível (Feigenbaum, 1994).

Outra definição de qualidade é, a proposta por Juran (2009), no sentido de desempenho do produto, que resulta de características que proporcionam a satisfação com o produto, levando os consumidores a comprá-lo. A qualidade pode representar a ausência de deficiências que, caso ocorresse, implicaria em insatisfação com o produto e reclamações de clientes (Juran, 2009).

Na visão de Sakurai (1997), há três interpretações do que constitui a qualidade: grau de conformidade, adequação ao uso e excelência inata. O grau de conformidade representa o produto que está conforme suas especificações. A adequação ao uso é voltada para o consumidor, pois a qualidade está ligada em atender à expectativa dele. E a excelência inata define a qualidade como uma característica natural da superioridade de um produto ou serviço.

A qualidade de um produto ou serviço pode ser a combinação de características referentes a marketing, engenharia, produção e manutenção para que os produtos ou serviços correspondam às expectativas do cliente (Feigenbaum, 1994). Feigenbaum (1994) menciona que a qualidade satisfatória de um produto ou processo expressa utilização satisfatória dos recursos e, consequentemente, custos reduzidos. E ao contrário, a qualidade insatisfatória é resultado da utilização insatisfatória dos recursos, o que provoca desperdícios de material, tempo de mão de obra e equipamentos.

Logo, com maior qualidade, os custos diminuem devido a menor quantidade de erros, atrasos, defeitos e reparos, além de utilizar melhor as máquinas e os materiais. $E$ 
consequentemente aumenta a produtividade por intermédio da captura do mercado com produtos de melhor qualidade e menores preços, o que garante a lucratividade e longevidade da empresa (Deming, 2003).

De acordo com Garvin (2002), as diferentes conceituações de qualidade classificam-se sob cinco principais abordagens: transcendente, pois a qualidade é reconhecida como excelência inata; baseada no produto, quando a qualidade é uma variável precisa e mensurável; baseada no usuário, quando busca atender as diferentes necessidades dos consumidores; baseada na produção, pois identifica a qualidade como conformidade com as especificações; e por fim, a abordagem baseada no valor, a qual ressalta que a qualidade deve ser percebida em relação ao preço do produto.

Feigenbaum (1994) apresenta que os Custos da Qualidade (CQ) podem ser classificados em custos do controle e custos provenientes de falha no controle. Os custos do controle são divididos em duas partes, custos de prevenção, que impedem as não conformidades e compreendem os gastos com a qualidade; e os custos de avaliação, que abrangem custos de manutenção da qualidade da organização por meio de análises formais dos produtos.

Os custos provenientes de falha no controle são causados por materiais e produtos que não atendem às especificações. São divididos em custos de falhas internas, que incluem custos ocorridos quando o produto está dentro da organização, como material refugado, danificado e retrabalhado; e custos das falhas externas, que incluem custos situados na parte externa da organização, como falhas provenientes do desempenho do produto e reclamações dos clientes (Feigenbaum, 1994). A Figura 1 apresenta a classificação dos custos da qualidade. 


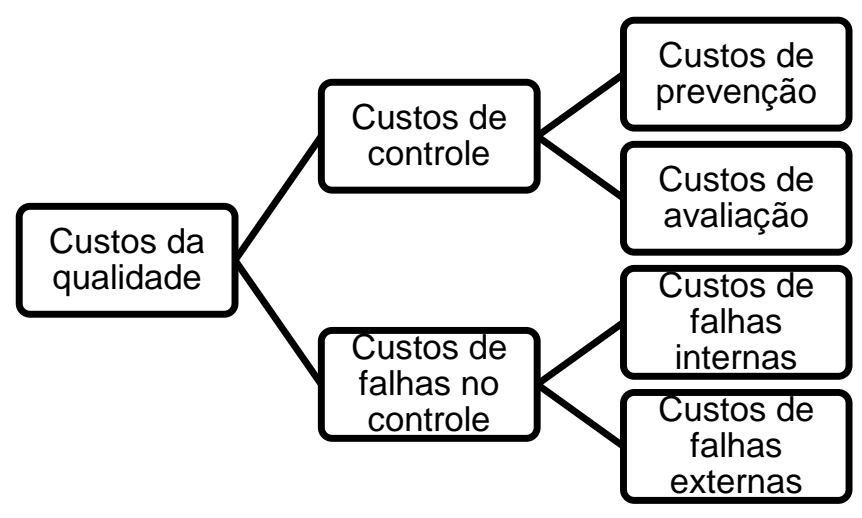

Figura 1. Classificação dos custos da qualidade

Fonte: Feigenbaum, 1994.

Os custos de prevenção e avaliação objetivam um maior nível de qualidade, ou seja, uma curva de inclinação positiva com relação ao nível de qualidade. Por outro lado, os custos de falhas internas e externas refletem uma curva de inclinação negativa contra o nível de qualidade, ou seja, quando mais baixo o nível de qualidade, maior será o custo das falhas do produto (Shank \& Govindarajan, 1997).

Assim, o somatório do custo total da qualidade origina uma curva em forma de "U" refletindo o contraste dos custos de prevenção e avaliação e custos de falhas, conforme evidenciado na Figura 2. 


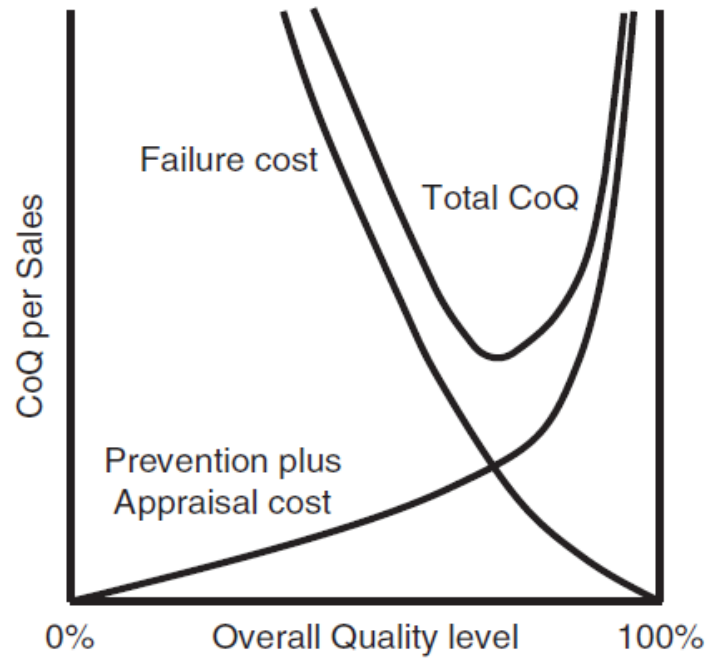

Figura 2. Modelo de Custos da Qualidade

Fonte: Schiffauerova e Thomson (2006).

É possível verificar na Figura 2 que quanto maior os investimentos em custos de prevenção e avaliação, menores são os custos com falhas internas e externas, sendo ideal encontrar um ponto "ótimo" de custos, o qual reflete o menor custo total da qualidade. Desse modo, o conceito de uma eficaz gestão estratégica de qualidade expressa à escolha do nível de qualidade e a composição de gastos entre as quatro categorias que minimize o custo total da qualidade (Shank \& Govindarajan, 1997).

\subsection{Estudos Relacionados}

Schiffauerova e Thomson (2006) analisaram a literatura sobre os CQ a fim de proporcionar uma melhor compreensão do tema e verificaram que organizações que utilizam programas de $C Q$ têm sido bem-sucedidas na redução dos custos e na melhoria da qualidade. Segundo os pesquisadores, o modelo mais utilizado nas organizações é o PAF (Prevenção, Avaliação e Falhas) de Feigenbaum.

Já Dale e Wan (2002) avaliaram o mais adequado modelo de CQ em uma organização de alimentos e bebidas. A principal constatação é que o método escolhido deve atender a situação da organização, baseando-se no conceito de melhoria 
Influência dos Custos de Controle nos Custos das falhas Internas e Externas Juliane Andressa Pavão, Simone Bernardes Voese, Reinaldo Rodrigues Camacho

contínua, deve ser aplicável a todos os departamentos e empregam uma abordagem de equipe. De acordo com Dale e Wan (2002), o sucesso do modelo de CQ dependerá da cultura e da disciplina da organização para seguir os procedimentos necessários.

Maldaner (2003) constatou em uma empresa de construção civil no Brasil que há uma grande falha na mensuração dos custos reais de produção e na identificação das falhas que ocorrem nos processos, apesar do grande investimento por parte dessas organizações em programas de qualidade.

Morgan e Ramos (2008) verificaram a inexistência o gerenciamento do CQ em $61 \%$ dos casos pesquisados no setor de construção civil, apesar dos respondentes considerarem o CQ útil para a gestão e serem certificadas no nível "A" do Programa Brasileiro da Qualidade e Produtividade do Habitat (PBQP-H).

Da mesma forma, Luz (2011) identificou em empresas do setor de construção civil que a maioria dos gestores adota conceitualmente os $C Q$, porém não mensuram esses custos, sendo que somente quatro gestores elaboram os relatórios dos custos da qualidade pelo sistema contábil.

Alencar e Guerreiro (2004) investigaram empresas finalistas do Prêmio Nacional de Qualidade visando identificar o desenvolvimento da mensuração dos resultados decorrentes da implantação dos programas de qualidade. Os pesquisadores verificaram que os resultados são calculados apenas parcialmente pelas organizações.

Souza e Collaziol (2006) também investigaram empresas integrantes do cadastro da Fundação para o Prêmio Nacional da Qualidade (FPNQ) e identificaram que a maioria das organizações pesquisadas não utiliza a classificação desenvolvida por Feigenbaum, sendo que somente $5,7 \%$ adotam relatórios segregando os $C Q$.

Já Mattos e Toledo (1998) afirmam que 39\% das empresas investigadas informaram ter um sistema de CQ implantado ou em implantação e que as dificuldades para implantação concentram-se no desconhecimento da ferramenta e na visão de que o sistema de informação contábil existente não é adequado.

Sá (2003) identificou nas indústrias de transformação os tratamentos dispensados aos $C Q$ e verificou que há maior incidência dos custos de falhas internas e 
Influência dos Custos de Controle nos Custos das falhas Internas e Externas Juliane Andressa Pavão, Simone Bernardes Voese, Reinaldo Rodrigues Camacho

externas. Achado esse de acordo com Plewa, Kaiser e Hartmann (2016) uma vez que os custos de falhas correspondem a aproximadamente $50 \%$ do total de CQ.

Em adição, Abdelsalam e Gad (2009) afirmam que os custos de falhas são os mais difíceis de mensurar, já que não há um registro específico mantido para os custos associados ao reparo de defeitos de qualidade. E que no setor de construção civil, os empreiteiros tentam esconder esses custos e não registram, porque têm medo de que, destacando os custos gastos para reparar os defeitos, sua imagem de bons empreiteiros possa se deteriorar diante de sua administração.

Guinot, Evans e Badar (2016) investigaram o impacto dos custos de qualidade no valor presente de um lançamento de um novo produto em uma montadora de automóveis norte-americana. O estudo mostrou que os $\mathrm{CQ}$ pode ter um impacto significativo em uma análise de valor presente e a gestão dos CQ devem ser consideradas durante o planejamento de pré-lançamento de um novo produto.

Ertel, Raupp e Borba (2017) tiveram como objetivo identificar os custos da qualidade e da não qualidade em uma indústria do segmento têxtil. Os resultados apontaram a ocorrência expressiva de custos com falhas internas e externas, principalmente de falhas internas. Os custos da não qualidade são superiores aos custos da qualidade, ou seja, a empresa está remediando situações ao invés de prevenir as mesmas.

Čermáková e Bris (2017) implementaram em uma empresa de manufatura o modelo PAF, que demonstrou sucesso em relação ao custo de qualidade para o período de 2010 a 2014. Segundo os pesquisadores, ocorreu uma potencial redução dos custos na categoria de falha interna. A empresa foi aconselhada a começar a gerenciar essa categoria e tentar gradualmente eliminar todas as causas de falhas internas.

\subsection{Hipóteses teórico-empírica da pesquisa}

Plewa, Kaiser e Hartmann (2016) avaliaram os CQ em 286 empresas de manufaturas alemãs e os pesquisadores concluíram que baixos valores de custos de 
falhas estão associados com altos valores de custos de prevenção e avaliação, o que confirma o pressuposto pela literatura. Porém, os pesquisadores identificaram, que ao contrário do que é apresentado na Curva de Custo Total da Qualidade, onde essa teria um formato em "U", nos dados investigados, depois de alcançar um determinado nível ótimo de qualidade, além da redução dos custos de falhas, também ocorre uma redução nos custos de prevenção e avaliação, conforme evidenciado na Figura 3.

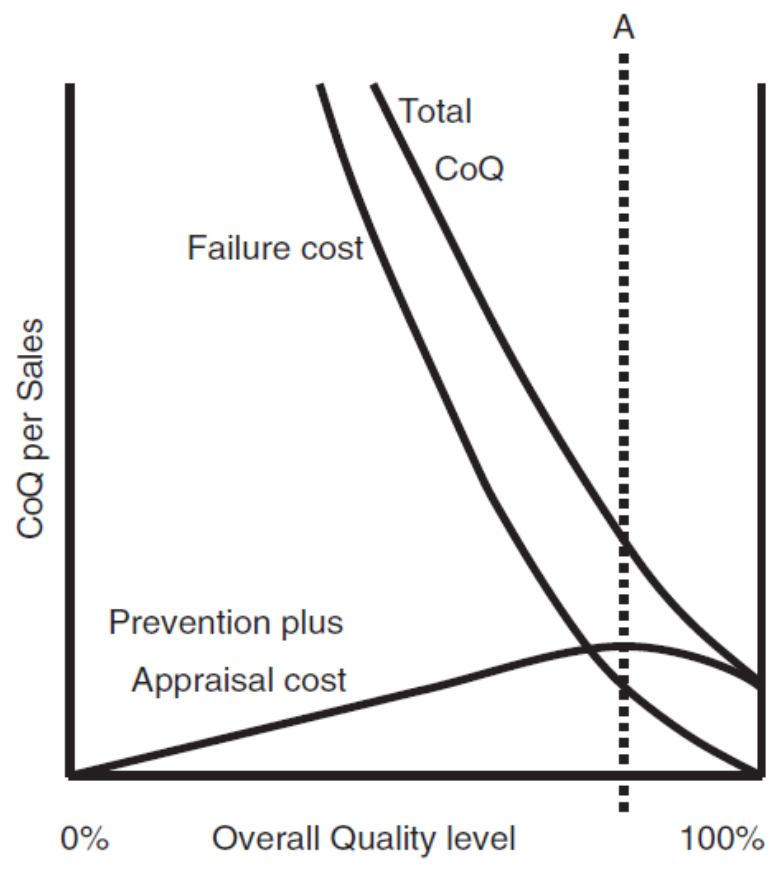

Figura 3. Modificação do modelo moderno

Fonte: Plewa, Kaiser e Hartmann (2016, p. 1280).

Abdelsalam e Gad (2009) avaliaram o CQ na construção residencial em Dubai e ainda testaram relações entre diferentes variáveis que afetam a CQ. Os achados mostram a existência de uma correlação positiva entre os custos (prevenção e avaliação) e os custos das falhas. Esses achados contestam a literatura dos $C Q$, uma vez que é esperada uma correlação negativa, ou seja, investindo em prevenção e avaliação almeja-se a redução das falhas. 
A fim de auxiliar a elucidar essa contraposição, apresentam-se as hipóteses teórico-empírica da pesquisa:

H1 - A ocorrência de custos com treinamento de pessoal influencia em menores custos de falhas internas e externas.

H2 - A ocorrência de custos com manutenção preventiva de equipamentos influencia em menores custos de falhas internas e externas.

H3 - A ocorrência de custos com desenvolvimento de sistema de qualidade influencia em menores custos de falhas internas e externas.

H4 - A ocorrência de custos com desenvolvimento de peça-piloto/moldes dos produtos influencia em menores custos de falhas internas e externas.

H5 - A ocorrência de custos com auditoria do sistema de qualidade influencia em menores custos de falhas internas e externas.

H6 - A ocorrência de custos com inspeção nos materiais comprados influencia em menores custos de falhas internas e externas.

H7 - A ocorrência de custos com inspeção nos produtos fabricados influencia em menores custos de falhas internas e externas.

H8 - A ocorrência de custos com avaliação dos produtos dos concorrentes influencia em menores custos de falhas internas e externas.

H9 - A ocorrência de custos com avaliação de conservação de materiais em estoque influencia em menores custos de falhas internas e externas.

\section{PROCEDIMENTOS METODOLÓGICOS}

Nessa seção são expostos o caminho percorrido para desenvolvimento da pesquisa. Conforme destaca Marconi e Lakatos (2003), o método é um conjunto de atividades que permite, com maior segurança, alcançar o objetivo, traçando o caminho e detectando erros.

A classificação da pesquisa, com relação à abordagem do problema, classificase como quantitativa, uma vez que esse método "caracteriza-se pelo emprego da 
quantificação tanto nas modalidades de coleta de informações, quanto no tratamento delas por meio de técnicas estatísticas" (Richardson, 2015, p. 70). Trata-se de um estudo descritivo, que teve como estratégia de pesquisa o levantamento do tipo survey e coleta de dados por meio de questionário.

O questionário possui 5 blocos de perguntas organizados a fim de facilitar o entendimento do respondente. No primeiro e segundo bloco foram questionados aspectos do perfil do respondente e da empresa respectivamente com perguntas abertas e fechadas. Os três últimos blocos questionam a ocorrência dos custos de prevenção, avaliação e de falhas em uma escala de 11 pontos, sendo 0 não ocorre, 1 ocorre pouco e até 10 ocorre muito. A Tabela 1 a seguir apresenta os constructos, variáveis do estudo e referências utilizadas.

Tabela 1

Constructos, variáveis e referências

\begin{tabular}{lcc}
\hline Constructos & Variáveis & Referências \\
\hline Custos de Prevenção & Treinamento de pessoal & \\
& Manutenção preventiva de equipamentos & \\
& Desenvolvimento de sistema de qualidade & \\
& Desenvolvimento de peça-piloto/moldes dos produtos & Almeida \\
Custos de Avaliação & Auditoria do sistema de qualidade & $(2011)$, \\
& Inspeção nos materiais comprados & Collaziol \\
& Inspeção nos produtos fabricados & $(2006)$, Robles \\
Custos de Falhas & Avaliação dos produtos dos concorrentes & $(2003)$ e Sá \\
Internas e Externas & Avaliação de conservação de materiais em estoque & $(2003)$ \\
& Retrabalho, sobra de materiais e retalhos, horas extras & \\
& para recuperar atrasos, tempo perdido devido à compra & \\
& de materiais defeituosos, vendas perdidas, devolução de & \\
& produto defeituoso e substituição do produto defeituoso & \\
& recusado pelo cliente. & \\
\hline
\end{tabular}

Nota. Fonte: a pesquisa, 2018.

Foi realizado o pré-teste do questionário inicialmente com três especialistas da contabilidade. Em seguida, o questionário foi testado em duas empresas com características da população. O tempo para resposta do questionário foi de dez minutos. 
Influência dos Custos de Controle nos Custos das falhas Internas e Externas Juliane Andressa Pavão, Simone Bernardes Voese, Reinaldo Rodrigues Camacho

Caracterizam-se como população deste estudo as indústrias de confecções associadas ao Sindicato da Indústria do Vestuário de Maringá (SINDVEST), Associação Comercial de Maringá (ACIM) e Sindicato da Indústria do Vestuário de Cianorte (SINVESTE), totalizando 252 empresas.

A coleta de dados ocorreu no período de outubro e dezembro de 2015. Primeiramente, entrou-se em contato por telefone com as empresas, visando à apresentação da pesquisa. Em seguida foi agendada uma visita para aplicação do questionário com a presença do pesquisador. Obteve-se 54 questionários respondidos em Maringá e 67 no município de Cianorte, totalizando 121 respostas, sendo que esse número atingiu o tamanho da amostra mínima com erro amostral de 10\% para os dois municípios investigados.

A fim de cumprir o objetivo proposto e testar as hipóteses do estudo, os dados foram analisados por meio de Análise Fatorial Exploratória (AFE) e Regressão Linear Múltipla com bootstraping e utilizou-se o software IBM Statistical Package for the Social Sciences (SPSS) versão 24. Primeiramente, empregou-se a AFE para reduzir a um único fator os indicadores de custos de falhas, o qual obteve uma Medida KaiserMeyer-Olkin no valor de 0,772 e Teste de esfericidade de Bartlett de 0,000 o que indica a AFE ser adequada. Em seguida, por meio da regressão múltipla, verificou-se a influência ou não das variáveis de custos de prevenção e avaliação na redução dos custos de falhas.

\section{APRESENTAÇÃO DOS RESULTADOS}

Os respondentes do questionário foram proprietários e gerentes das indústrias de confecções pertencentes ao Arranjo Produtivo Local (APL) dos municípios de Maringá e Cianorte, no Paraná. Dos respondentes 54\% são do gênero feminino e $40 \%$ possuem graduação. Aproximadamente $24 \%$ das empresas possuem até 5 anos de constituição e 94\% possuem menos de 100 funcionários. 
Influência dos Custos de Controle nos Custos das falhas Internas e Externas

Juliane Andressa Pavão, Simone Bernardes Voese, Reinaldo Rodrigues Camacho

A Tabela 2 apresenta as estatísticas descritivas para as variáveis analisadas. Verifica-se a normalidade dos dados por meio da assimetria e curtose, uma vez que os valores da assimetria ficam entre -3 e +3 e da curtose entre -10 e +10 (Marôco, 2010).

Tabela 2

Estatísticas descritivas das variáveis

\begin{tabular}{|c|c|c|c|c|c|c|c|c|c|}
\hline & & $\mathbf{N}$ & $\begin{array}{l}\text { Míni- } \\
\text { mo }\end{array}$ & $\begin{array}{l}\text { Máxi- } \\
\text { mo }\end{array}$ & $\begin{array}{l}\text { Mé- } \\
\text { dia }\end{array}$ & $\begin{array}{l}\text { Medi- } \\
\text { ana }\end{array}$ & $\begin{array}{l}\text { Des- } \\
\text { vio Pa- } \\
\text { drão }\end{array}$ & $\begin{array}{l}\text { Assi- } \\
\text { metria }\end{array}$ & $\begin{array}{c}\text { Curto } \\
\text { se }\end{array}$ \\
\hline \multirow{5}{*}{ 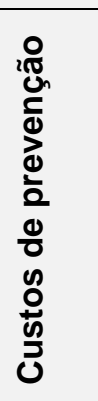 } & Treinamento de pessoal & 121 & 0 & 10 & 4,83 & 5,0 & 3,174 & $-0,098$ & $-1,101$ \\
\hline & $\begin{array}{l}\text { Manutenção preventiva } \\
\text { dos equipamentos }\end{array}$ & 121 & 0 & 10 & 6,88 & 8,0 & 3,042 & $-0,912$ & $-0,283$ \\
\hline & $\begin{array}{l}\text { Desenvolvimento de } \\
\text { sistema de qualidade }\end{array}$ & 121 & 0 & 10 & 5,74 & 7,0 & 3,285 & $-0,472$ & $-1,010$ \\
\hline & $\begin{array}{l}\text { Desenvolvimento de } \\
\text { peça-piloto/moldes dos } \\
\text { produtos }\end{array}$ & 121 & 0 & 10 & 8,75 & 10,0 & 2,567 & $-2,504$ & 5,424 \\
\hline & $\begin{array}{l}\text { Auditoria do sistema de } \\
\text { qualidade }\end{array}$ & 121 & 0 & 10 & 4,58 & 5,0 & 3,714 & 0,059 & $-1,535$ \\
\hline \multirow{4}{*}{ 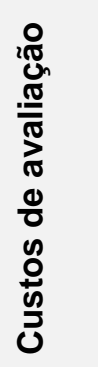 } & $\begin{array}{l}\text { Inspeção nos materiais } \\
\text { comprados }\end{array}$ & 121 & 0 & 10 & 7,74 & 8,0 & 2,574 & $-1,201$ & 0,708 \\
\hline & $\begin{array}{l}\text { Inspeção nos produtos } \\
\text { fabricados }\end{array}$ & 121 & 1 & 10 & 8,95 & 10,0 & 1,779 & $-2,209$ & 5,302 \\
\hline & $\begin{array}{l}\text { Avaliação dos produtos } \\
\text { dos concorrentes }\end{array}$ & 121 & 0 & 10 & 5,47 & 6,0 & 3,104 & $-0,411$ & $-0,781$ \\
\hline & $\begin{array}{l}\text { Avaliação de } \\
\text { conservação de } \\
\text { materiais em estoque }\end{array}$ & 121 & 0 & 10 & 7,85 & 9,0 & 2,682 & $-1,625$ & 2,203 \\
\hline \multirow{8}{*}{ 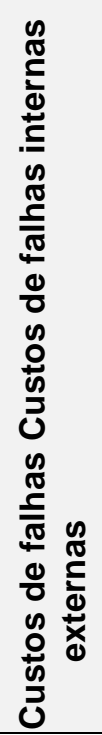 } & Retrabalho & 121 & 0 & 10 & 3,92 & 3,0 & 2,77 & 0,579 & $-0,653$ \\
\hline & $\begin{array}{l}\text { Sobra de materiais e } \\
\text { retalhos }\end{array}$ & 121 & 0 & 10 & 3,88 & 3,0 & 2,92 & 0,690 & $-0,543$ \\
\hline & $\begin{array}{l}\text { Horas extras para } \\
\text { recuperar atrasos }\end{array}$ & 121 & 0 & 10 & 2,68 & 1,0 & 3,08 & 1,076 & $-0,053$ \\
\hline & $\begin{array}{l}\text { Tempo perdido devido a } \\
\text { compra de materiais } \\
\text { defeituosos }\end{array}$ & 121 & 0 & 10 & 2,34 & 2,0 & 2,33 & 1,242 & 1,457 \\
\hline & $\begin{array}{l}\text { Descontos no preço de } \\
\text { venda de produtos com } \\
\text { defeitos }\end{array}$ & 121 & 0 & 10 & 2,99 & 2,0 & 3,09 & 0,963 & $-0,320$ \\
\hline & $\begin{array}{l}\text { Vendas perdidas devido } \\
\text { a baixa qualidade }\end{array}$ & 121 & 0 & 10 & 2,14 & 1,0 & 2,47 & 1,426 & 1,647 \\
\hline & $\begin{array}{l}\text { Devolução de produto } \\
\text { defeituoso }\end{array}$ & 121 & 0 & 10 & 1,93 & 1,0 & 2,26 & 1,755 & 2,994 \\
\hline & $\begin{array}{l}\text { Substituição do produto } \\
\text { defeituoso recusado } \\
\text { pelo cliente }\end{array}$ & 121 & 0 & 10 & 2,08 & 1,0 & 2,40 & 1,942 & 3,455 \\
\hline
\end{tabular}

Nota. Fonte: a pesquisa, 2018. 
Foram analisados os pressupostos para análise da regressão. A homocedasticidade dos dados foi verificada por meio do gráfico de dispersão, não sendo detectados dados heterocedásticos. A independência dos erros foi analisada por meio do Teste de Durbin-Watson que apresentou valor igual a 1,939, valor esse bem próximo de 2, o que representa ser adequado para a realização da regressão. Não foram identificados outliers pela Distância de Mahalanobis. As variáveis não apresentam Multicolinearidade, o que pode ser observado na Tabela 3 por meio da Matriz de Correlação de Pearson. As variáveis independentes não possuem forte correlação entre si, todos os valores encontram-se abaixo de 0,70 , portanto, não há multicolinearidade entre as variáveis. 
Influência dos Custos de Controle nos Custos das falhas Internas e Externas

Juliane Andressa Pavão, Simone Bernardes Voese, Reinaldo Rodrigues Camacho

Tabela 3

Matriz de Correlação de Pearson

\begin{tabular}{|c|c|c|c|c|c|c|c|c|c|c|c|}
\hline & & CPR1 & CPR2 & CPR3 & CPR4 & CPR5 & CAV1 & CAV2 & CAV3 & CAV4 & $\begin{array}{l}\text { CFA- } \\
\text { LHAS }\end{array}$ \\
\hline CPR1 & $\begin{array}{l}\text { Treinamento de } \\
\text { pessoal }\end{array}$ & 1 & & & & & & & & & \\
\hline CPR2 & $\begin{array}{l}\text { Manutenção } \\
\text { preventiva dos } \\
\text { equipamentos }\end{array}$ & ,446** & 1 & & & & & & & & \\
\hline CPR3 & $\begin{array}{l}\text { Desenvolvimento de } \\
\text { sistema de qualidade }\end{array}$ &, $461^{* *}$ &, $518^{* *}$ & 1 & & & & & & & \\
\hline CPR4 & $\begin{array}{l}\text { Desenvolvimento de } \\
\text { peça-piloto/moldes } \\
\text { dos produtos }\end{array}$ & ,230" & ,489** & , 448, & 1 & & & & & & \\
\hline CPR5 & $\begin{array}{l}\text { Auditoria do sistema } \\
\text { de qualidade }\end{array}$ &, $511^{* *}$ & , $491^{* *}$ & ,664 & ,399** & 1 & & & & & \\
\hline CAV1 & $\begin{array}{l}\text { Inspeção nos } \\
\text { materiais comprados }\end{array}$ & ,242** & , 335 & , $362^{* *}$ & , 217 & , $351^{* *}$ & 1 & & & & \\
\hline CAV2 & $\begin{array}{l}\text { Inspeção nos produtos } \\
\text { fabricados }\end{array}$ & 241* & , 307 &, $313^{* *}$ & 2222* &, $297^{* *}$ &, $554^{* *}$ & 1 & & & \\
\hline CAV3 & $\begin{array}{l}\text { Avaliação dos } \\
\text { produtos dos } \\
\text { concorrentes }\end{array}$ & 247* & 0,125 & "242, & 0,109 & 279" & 0,162 & 0,086 & 1 & & \\
\hline CAV4 & $\begin{array}{l}\text { Avaliação de } \\
\text { conservação de } \\
\text { materiais em estoque }\end{array}$ & (189* & , $412^{* *}$ & "266" & ,326* & ,439** &, $563^{* *}$ & , $461^{* *}$ & ,272** & 1 & \\
\hline $\begin{array}{l}\text { CFA- } \\
\text { LHAS }\end{array}$ & $\begin{array}{l}\text { Custos de falhas } \\
\text { internas e externas }\end{array}$ & ,738** &, $523^{* *}$ & ,656" & , 326** & ,640** & 0,089 & 0,165 & 0,138 & 0,073 & 1 \\
\hline
\end{tabular}

Nota. Fonte: a pesquisa, 2018.

${ }^{* *}$ A correlação é significativa no nível 0,01 (bilateral).

* A correlação é significativa no nível 0,05 (bilateral).

O modelo apresentou grande significância estatística $(p=0,000)$ e obteve um $R^{2}$ de 0,77 . Ou seja, $77 \%$ da variância da ocorrência dos custos de falhas são explicadas nesse modelo. Para validação interna do modelo de regressão linear múltipla foi efetuada a técnica bootstrap baseado em 1000 replicações. Assim, relizou-se o bootstrap com 1000 amostras determinadas por amostragem simples e intervalo de confiança de 95\%. A Tabela 4 mostra os coeficientes, erros e a significância das hipóteses testadas. 
Influência dos Custos de Controle nos Custos das falhas Internas e Externas

Juliane Andressa Pavão, Simone Bernardes Voese, Reinaldo Rodrigues Camacho

Tabela 4

Modelo original e Bootstrap da Regressão Linear Múltipla

\begin{tabular}{|c|c|c|c|c|c|c|c|c|c|}
\hline & & \multicolumn{4}{|c|}{ Modelo original } & \multicolumn{3}{|c|}{ Bootstrap } & \multirow[b]{2}{*}{$\begin{array}{c}\text { Hipótese } \\
\text { Rejeitada } \\
\text { ou não }\end{array}$} \\
\hline & & $\begin{array}{l}\text { Coeficiente } \\
\text { não } \\
\text { padronizado } \\
\text { Beta } \\
\end{array}$ & $\begin{array}{c}\text { Erro } \\
\text { Padrão }\end{array}$ & $\begin{array}{c}\text { Coeficiente } \\
\text { padronizado } \\
\text { Beta }\end{array}$ & Sig. & $\begin{array}{l}\text { Coeficiente } \\
\text { Beta }\end{array}$ & $\begin{array}{c}\text { Erro } \\
\text { Padrão }\end{array}$ & Sig. & \\
\hline & (Constante) & $-0,818$ & 0,272 & & & $-0,818$ & 0,286 & $\begin{array}{c}0,00 \\
5^{\star}\end{array}$ & \\
\hline H1 & $\begin{array}{l}\text { Treinamento } \\
\text { de pessoal }\end{array}$ & 0,152 & 0,018 & 0,484 & $\begin{array}{c}0,00 \\
0^{\star}\end{array}$ & 0,152 & 0,018 & $\begin{array}{c}0,00 \\
1^{*}\end{array}$ & $\begin{array}{c}\text { Não } \\
\text { rejeitada }\end{array}$ \\
\hline $\mathrm{H} 2$ & $\begin{array}{l}\text { Manutenção } \\
\text { preventiva } \\
\text { dos } \\
\text { equipamentos }\end{array}$ & 0,051 & 0,020 & 0,155 & $\begin{array}{c}0,01 \\
3^{\star}\end{array}$ & 0,051 & 0,019 & $\begin{array}{c}0,01 \\
1^{*}\end{array}$ & $\begin{array}{l}\text { Não } \\
\text { rejeitada }\end{array}$ \\
\hline H3 & $\begin{array}{l}\text { Desenvolvime } \\
\text { nto de } \\
\text { sistema de } \\
\text { qualidade }\end{array}$ & 0,091 & 0,021 & 0,299 & $\begin{array}{c}0,00 \\
0^{*}\end{array}$ & 0,091 & 0,022 & $\begin{array}{c}0,00 \\
1^{*}\end{array}$ & $\begin{array}{c}\text { Não } \\
\text { rejeitada }\end{array}$ \\
\hline H4 & $\begin{array}{l}\text { Desenvolvime } \\
\text { nto de peça- } \\
\text { piloto/moldes } \\
\text { dos produtos }\end{array}$ & $-0,003$ & 0,021 & $-0,007$ & $\begin{array}{c}0,90 \\
6\end{array}$ & $-0,003$ & 0,026 & $\begin{array}{c}0,92 \\
4\end{array}$ & Rejeitada \\
\hline H5 & $\begin{array}{l}\text { Auditoria do } \\
\text { sistema de } \\
\text { qualidade }\end{array}$ & 0,074 & 0,019 & 0,274 & $\begin{array}{c}0,00 \\
0^{*}\end{array}$ & 0,074 & 0,020 & $\begin{array}{c}0,00 \\
3^{*}\end{array}$ & $\begin{array}{l}\text { Não } \\
\text { rejeitada }\end{array}$ \\
\hline H6 & $\begin{array}{l}\text { Inspeção nos } \\
\text { materiais } \\
\text { comprados }\end{array}$ & $-0,073$ & 0,024 & $-0,187$ & $\begin{array}{c}0,00 \\
3^{*}\end{array}$ & $-0,073$ & 0,018 & $\begin{array}{c}0,00 \\
1^{*}\end{array}$ & $\begin{array}{l}\text { Não } \\
\text { rejeitada }\end{array}$ \\
\hline H7 & $\begin{array}{l}\text { Inspeção nos } \\
\text { produtos } \\
\text { fabricados }\end{array}$ & 0,006 & 0,032 & 0,011 & $\begin{array}{c}0,85 \\
1\end{array}$ & 0,006 & 0,033 & $\begin{array}{c}0,86 \\
2\end{array}$ & Rejeitada \\
\hline H8 & $\begin{array}{l}\text { Avaliação dos } \\
\text { produtos dos } \\
\text { concorrentes }\end{array}$ & $-0,025$ & 0,016 & $-0,077$ & $\begin{array}{c}0,12 \\
2\end{array}$ & $-0,025$ & 0,019 & $\begin{array}{c}0,18 \\
4\end{array}$ & Rejeitada \\
\hline H9 & $\begin{array}{l}\text { Avaliação de } \\
\text { conservação } \\
\text { de materiais } \\
\text { em estoque }\end{array}$ & $-0,059$ & 0,024 & $-0,158$ & $\begin{array}{c}0,01 \\
5^{\star}\end{array}$ & $-0,059$ & 0,023 & $\begin{array}{c}0,00 \\
8^{\star}\end{array}$ & $\begin{array}{l}\text { Não } \\
\text { rejeitada }\end{array}$ \\
\hline
\end{tabular}

Nota. Fonte: a pesquisa, 2018.

Percebe-se na Tabela 4 que os valores dos coeficientes, o erro padrão e os valores de significância estatística dos valores obtidos para as amostras bootstrap são muito próximos dos valores obtidos na amostra original, o que evidencia a validade do modelo de regressão. 
Influência dos Custos de Controle nos Custos das falhas Internas e Externas Juliane Andressa Pavão, Simone Bernardes Voese, Reinaldo Rodrigues Camacho

Com relação aos custos de prevenção, a ocorrência de treinamento de pessoal (H1) influencia $48 \%(p=0,000)$ na redução de custos de falhas das empresas investigadas do setor de confecções. O que se deve pelo fato da costura ser uma das principais atividades do processo produtivo, sendo essencial um funcionário treinado para evitar defeitos nas peças.

A manutenção preventiva de equipamentos (H2) também se mostrou com significância estatística ( $p=0,013)$, uma vez que manutenção das máquinas de corte e costura influencia em $15 \%$ na redução de custos de falhas, como atraso no processo produtivo, bem como a produção de peças com defeitos decorrentes das máquinas.

$O$ desenvolvimento de sistema de qualidade $(H 3)(p=0,000)$ e a auditoria do sistema de qualidade $(\mathrm{H} 5) \quad(p=0,000)$ apresentaram forte significância estatística influenciando a redução na ocorrência dos custos de falhas em aproximadamente 30\% e $27 \%$ respectivamente. Isso mostra o importante papel do sistema de qualidade nas empresas de confecções a fim da melhoria dos processos e produtos e, consequente, redução dos custos de falhas.

Com relação aos custos de prevenção, apenas o desenvolvimento de peçaspiloto e moldes não influenciou na ocorrência dos custos de falhas, sendo a $\mathrm{H} 4$ rejeitada. Dessa forma, percebe-se que investir em custos de prevenção reduz os custos de falhas, assim como pressupõe a literatura, ou seja, é melhor prevenir do que remediar. Achados esses em consonância em parte com a pesquisa de Plewa, Kaiser e Hartmann (2016) em que observaram que baixos valores de custos de falhas estão associados com altos valores de custos de prevenção em empresas de manufaturas.

No que se refere aos custos de avaliação, a inspeção de materiais comprados (H6) $(p=0,003)$ e a avaliação da conservação de materiais em estoque (H9) $(p=0,015)$ apresentaram um coeficiente negativo, o que representa a relação inversa. Ou seja, a inspeção de materiais comprados e a avaliação da conservação de materiais em estoque influenciam em $18 \%$ e $15 \%$ para a ocorrência de custos de falhas respectivamente nas empresas do setor de confecções estudadas, o que contraria a literatura, uma vez que investir em avaliação e inspeção deveria evitar a ocorrência de 
Influência dos Custos de Controle nos Custos das falhas Internas e Externas

Juliane Andressa Pavão, Simone Bernardes Voese, Reinaldo Rodrigues Camacho

falhas. Ao mesmo tempo, achado esse corrobora em parte com o estudo de Abdelsalam e Gad (2009) que observou uma correlação positiva em custos de avaliação e de falhas no setor de construção civil.

A inspeção em produtos fabricados (H7) não apresentou significância estatística, o que talvez seja consequência dos produtos fabricados não conter muitos defeitos tornando desnecessário a sua inspeção. A H8 também foi rejeitada, já que a avaliação dos produtos dos concorrentes não obteve significância estatística.

\section{CONSIDERAÇÕES FINAIS}

Este estudo teve como objetivo analisar a influência de custos de prevenção e avaliação na redução de custos de falhas em 121 empresas do setor de confecções nos municípios de Cianorte e Maringá, Estado do Paraná.

O principal achado desta pesquisa é que investir no treinamento de funcionários, na manutenção preventiva de máquinas e equipamentos, desenvolver e auditar um sistema de qualidade influencia na redução dos custos de falhas, ou seja, é melhor prevenir do que remediar. Porém, a inspeção de materiais comprados e a avaliação da conservação dos materiais em estoque apresentaram comportamento significativo contrário, ocasionando assim, os custos de falhas.

Resumindo, os custos de prevenção reduzem a ocorrência de custos de falhas, já os custos de avaliação apresentaram comportamento negativo na redução dos custos de falhas na amostra investigada. O que faz com que este estudo contribua com as pesquisas de Abdelsalam e Gad (2009) e Plewa, Kaiser e Hartmann (2016), demonstrando um achado distinto dos estudos realizados até o momento. Tendo como principal contribuição para a literatura a verificação de uma diferença existente entre os custos de prevenção e avaliação na ocorrência dos custos de falhas, sendo que esses custos, denominados de custos do controle, por Feigenbaum (1994) muitas vezes são tratados e analisados em conjunto. Assim, a implicação fundamental deste estudo é a 
Influência dos Custos de Controle nos Custos das falhas Internas e Externas

Juliane Andressa Pavão, Simone Bernardes Voese, Reinaldo Rodrigues Camacho

distinção entre os custos de prevenção e avaliação, que devem ser abordados de forma distinta, tanto na perspectiva teórica como prática.

Este estudo possui como limitações a amostra investigada por se caracterizar apenas empresas do setor de confecções e em sua maioria de micro e pequeno porte. As variáveis selecionadas também são uma limitação, bem como o fato de ser utilizada uma escala de ocorrência para os custos da qualidade, uma vez que não seria possível obter os valores monetários. Sugere-se para futuras pesquisas a investigação da relação entre os custos de prevenção, custos de avaliação e custos de falhas, e como a ocorrência de um pode afetar a ocorrência de outro.

\section{REFERÊNCIAS}

Abdelsalam, H. M., \& Gad, M. M. (2009). Cost of quality in Dubai: An analytical case study of residential construction projects. International journal of project management, 27(5), 501-511.

Alencar, R. C., \& Guerreiro, R. (2004). A mensuração do resultado da qualidade em empresas brasileiras. Revista Contabilidade \& Finanças-USP, 15, 7-23.

Carreira, S. da S. (2001). Análise dos fatores de sucesso das empresas no ramo de confecções na região noroeste do Paraná "Corredor da moda" Período 1990 a 2000. (Dissertação de Mestrado). Universidade Federal de Santa Catarina, UFSC, Florianópolis, SC, Brasil.

Čermáková, M., \& Bris, P. (2017). Managing the costs of quality in a Czech manufacturing company. Scientific papers of the University of Pardubice. Series D, Faculty of Economics and Administration. 41/2017.

Collaziol, E. (2006). Custos da qualidade: uma investigação da pratica e percepção empresarial. (Dissertação de Mestrado). Programa de Pós-Graduação em Ciências Contábeis, Universidade do Vale do Rio dos Sinos, Unisinos, São Leopoldo, RS, Brasil.

Crosby, P. B. (1979). Quality is free: The art of marketing quality certain. New York: New American Library. 
Dale, B. G., \& Wan, G. M. (2002). Setting up a quality costing system: an evaluation of the key issues. Business Process Management Journal, 8(2), 104-116.

Deming, W. E. (2003). Saia da crise. Rio de Janeiro: Futura.

Ertel, M. E. A., Raupp, F. M., \& de Borba, J. T. (2018). Custos da qualidade e da não qualidade no setor têxtil: um estudo de caso. FACEF Pesquisa-Desenvolvimento e Gestão, 20(2), 221-232.

Feigenbaum, A. V. (1994). Controle da qualidade total. Makron Books.

Garvin, D. A. (2002). Gerenciando a qualidade: a visão estratégica e competitiva. (J. F. B. de Souza, Trad.). Rio de Janeiro: Qualitymark.

Guinot, J., Evans, D., \& Badar, M. A. (2016). Cost of quality consideration following product launch in a present worth assessment. International Journal of Quality \& Reliability Management, 33(3), 399-413.

Juran, J. M. (2009). A qualidade desde o projeto: novos passos para o planejamento da qualidade em produtos e serviços. (N. Montingelli Jr., Trad.). São Paulo: Cengage Learning.

Juran, J. M., \& Gryna, F. M., Jr. (1970). Quality planning and analysis: from product development through usage. New Delhi: McGraw-Hill.

Lakatos, E. M., \& Marconi, M. de A. (2003). Fundamentos de metodologia científica. (5a ed.). São Paulo: Atlas.

Luz, J. R. M. (2011). Gestão estratégica baseada na qualidade e custos da qualidade: um estudo no setor de construção civil da cidade de Campina Grande-PB. Dissertação de Mestrado em Ciências Contábeis, Universidade de Brasília, Brasília.

Maldaner, S. M. (2003). Procedimento para identificação de custos da não-qualidade na construção civil. (Dissertação de Mestrado). Programa de Pós-Graduação em Engenharia de Produção, Universidade Federal de Santa Catarina, UFSC, SC, Brasil.

Marconi, M. de A., \& Lakatos, E. M. (2006). Técnicas de pesquisa: planejamento e execução de pesquisas, amostragens e técnicas de pesquisa, elaboração, análise e interpretação de dados. (6a ed.). São Paulo: Atlas. 
Mattos, J. C., \& de Toledo, J. C. (1999). Custos da qualidade: diagnóstico nas empresas com certificação ISO 9000. Revista de Administração da Universidade de São Paulo, 34(2).

Morgan, B. F., \& Ramos, L. L. (2008). Mensuração dos custos da qualidade nas empresas de construção civil. Enfoque: Reflexão Contábil, 27(3).

Plewa, M., Kaiser, G., \& Hartmann, E. (2016). Is quality still free? Empirical evidence on quality cost in modern manufacturing. International Journal of Quality \& Reliability Management, 33(9), 1270-1285.

Richardson, R. J. (2015). Pesquisa social: métodos e técnicas. (3a ed.). São Paulo: Atlas.

Sá, V. M. R. (2003). Custo da qualidade nas indústrias de transformação de Pernambuco. (Dissertação de Mestrado). Programa de Pós-Graduação em Engenharia de Produção, Universidade Federal de Pernambuco, UFPE, PE, Brasil.

Sakurai, M. (1997). Gerenciamento integrado de custos. São Paulo: Atlas.

Schiffauerova, A., \& Thomson, V. (2006). A review of research on cost of quality models and best practices. International Journal of Quality \& Reliability Management, 23(6), 647-669.

Shank, J. K., \& Govindarajan, V. (1997). A revolução dos custos. Rio de Janeiro: Campus.

Souza, M. A., \& Collaziol, E. (2006). Planejamento e controle dos custos da qualidade: Uma investigação da prática empresarial. Revista Contabilidade \& FinançasUSP, 17(41), 38-55.

Data de Submissão: 21/05/2020

Data de Aceite: $24 / 11 / 2020$ 